\title{
"Lucinde" de Friedrich Schlegel o la novela experimental del romanticismo temprano"
}

Friedrich Schlegel's “Lucinde”, or the Early Romanticism’s experimental novel

\author{
Gonzalo Portales
}

Instituto de Filosofía, Facultad de Filosofía y Humanidades, Universidad Austral de Chile.

Correo electrónico: gportale@uach.cl

El artículo intenta aclarar el estatus de novela experimental atribuido al texto Lucinde de Friedrich Schlegel, proponiendo una interpretación que lo sitúe dentro de los marcos generales de la propuesta estética del Romanticismo temprano alemán. Para ello desarrolla un análisis de su contenido teniendo como hilo conductor el programa filosófico-literario de la progressiveuniversal-Poesie que caracterizó a los autores de la así llamada Escuela de Jena.

Palabras clave: Literatura, romanticismo, filosofía

The article attempts to clarify the experimental novel status attributed to Friedrich Schlegel's opus Lucinde, advancing an interpretation that places it within the general framework of the aesthetic proposal of Early German Romanticism. In order to do this, it develops an analysis of the contents of Lucinde, following the thread of the progressive-universal-Poesie and its literary-philosophical program that characterized the authors of the so-called School of Jena.

Key words: Literature, romaticism, philosophy

“Cómo se puede escribir lo que apenas está permitido decir, lo que sólo se debiera sentir?"

Friedrich Schlegel, Lucinde.

Una de las particularidades del movimiento romántico que se identifica con la ciudad de Jena y con los escasos volúmenes que alcanzó a editar la revista Athenäeum, publicada entre los años 1798 y 1800, se manifiesta en el hecho de que aquellos que participaron de esta transformación estética, encabezada por el joven Friedrich Schlegel, intentaron cumplir a cabalidad la consigna de que ya habían arribado los tiempos en que se volvía imperioso superar la diferencia de los géneros literarios

Este trabajo es un resultado parcial del proyecto Fondecyt $n^{\circ} 1130533$. 
y enfrentar de manera crítica toda producción de escritura, independientemente de su procedencia, obviando así las fronteras impuestas por esa severa taxonomía que acostumbraba a separar historia de poesía y que se concedía, sin más, la facultad para clasificar la ficción como necesariamente ajena a la filosofía. Con ello se insinuaba, además, que se habían desvanecido también -al menos en el campo literario- las rígidas líneas divisorias que separaban lo sagrado de lo profano. A partir de este nuevo sincretismo, la teoría hermenéutica de Friedrich Schleiermacher podía proponer, no obstante las predisposiciones confesionales implicadas en su origen exegético bíblico, un método explicativo que no reconociera en la obra escrita la necesidad de doblegarse ante una autoridad pre-textual, pues, si bien se podía ser considerado y deferente con la tradición de la inspiratio, no por eso se admitiría ya que alguna escritura pudiese reclamar para sí el privilegio de eximirse de ser sometida al rigor analítico y crítico de una forma de interpretación que se concebía a sí misma como una doctrina del comprender. ${ }^{1}$

Luego de una muy intensa y ansiosa incursión por la literatura griega clásica en la que los escritos de Homero, Platón, Sófocles y Aristófanes son examinados a partir de los mismos parámetros de análisis y lectura-, el joven Friedrich Schlegel ${ }^{2}$ intenta alcanzar su propio tono poético mediante el cultivo del aforismo à la Lichtenberg y explora posibles caminos de emancipación estética proponiendo una innovadora reivindicación literaria del fragmento. Enaltecido por Goethe en sus Máximas y Reflexiones, el estilo de la escritura fragmentaria no sólo había dejado de ser considerado como una carencia de determinación y, por lo tanto, como un mero estado de inconclusión retórica, sino que se mostraba incluso como un modo privilegiado para combinar literatura y pensamiento en una sola expresión, acercando así poesía y filosofía hasta coincidencias anteriormente insospechadas.

La apertura a la novedad mueve a los jóvenes románticos a ensayar con amplia libertad propuestas escriturales en ámbitos diversos de la filosofía y de las artes. Bajo la premisa de romantizar el mundo se permiten sacar conclusiones sui generis no sólo de la Wissenchaftslehre de Fichte -filosofía reciente que se empinaba por sobre el criticismo kantiano- radicalizando así el concepto moderno de subjetividad, sino también de la literatura considerada en su formato futurista, es decir, de la novela. ${ }^{3}$

La categorización historiográfica ha usado ya desde hace varias décadas la expresión novelas experimentales (Romanexperimente) para referirse a algunos de estos intentos literarios producidos en lengua alemana durante el cambio de siglo entre el XVIII y el XIX-, ordenando bajo esta nomenclatura, la que busca constatar el nacimiento de una escritura capaz de correr riesgos al separarse conscientemente de

La expresión técnica de Schleiermacher es Kunstlehre des Verstehens, es decir una especie de ars intelligendi que se efectúa a partir de un procedimientos regido por una legislación que regulariza el acto interpretativo, liberándolo del pre-juicio y diferenciándolo de la mera opinión. En sus lecciones universitarias, August Wilhelm Schlegel no sólo había relativizado de manera radical el canon genérico de la literatura, sino que había puesto en duda también su periodización cronológica como criterio interpretativo. Cf. Holmes: 2006, p. 89 y ss.

2 Los trabajos de este período se encuentran reunidos en el primer tomo de la edición crítica de las obras de Friedrich Schlegel (= FSKA). Dilthey (Leben Schlaiermachers I, 1, p. 231) describe este modo de leer como aquel que corresponde más a los cambiantes intereses de un aficionado (Liebhaber) que se deja llevar por sus propias predilecciones, que a la exactitud y rigor metódico de un estudiante de filología.

Género literario que es resaltado en el romanticismo temprano -en los fragmentos del Atheneo- como la literatura que marcará las tendencias en el siglo XIX y que se identifica incluso etimológicamente con el movimiento, pues en alemán novela se dice, precisamente, Roman. 
formas ya largamente consagradas, a obras de procedencias tan heterogéneas como el Hyperion de Hölderlin, Heinrich von Ofterdingen de Novalis, Godwi de Clemens Brentano y Lucinde de Friedrich Schlegel (Cf. Schulz 1983: 398 y ss.).

Aun cuando parece conveniente adoptar sin reservas esta clasificación y continuar aplicando su criterio en la actualidad, habría que preguntarse, empero, por el sentido propio del concepto de experimento en relación al contenido de estos escritos. Especialmente la novela Lucinde, editada en Berlín el año 1799, ha sido objeto, ya inmediatamente después de su publicación y en los tiempos posteriores, de una apasionada discusión, la que no siempre se ha mantenido dentro de los marcos característicos de la recepción literaria habitual, sino que en ocasiones se extendió también al ámbito de la moralidad y de las así llamadas buenas costumbres (Zimmermann 2009: 136 y ss.; Behler 1996: 56 y ss.). Las imputaciones de libertinaje y cinismo dirigidas a la novela provocaron, sin embargo, una férrea defensa de la obra y de su autor por parte de los círculos literarios en torno al Salon berlinés de Henrriette Herz, cuya expresión paradigmática la constituyó la reseña anónima Vertraute Briefe über Friedrich Schlegels Lucinde, publicada en el volumen del mes de julio de 1800 de la revista Berlinisches Archiv der Zeit und ihres Geschmacks. ${ }^{4}$

El escándalo que caracteriza a parte de esta recepción polémica encuentra su principal causa, a mi juicio, en el hecho de que la novela no disimula su entusiasmo por cierto elogio de la sensibilidad corpórea, cuestión que parece haber herido hábitos y costumbres consuetudinarios al pudor académico-burgués en relación a la creación artística, y haber despertado así nuevamente su fobia a la obscenidad. Reacción que se produce sin percibir siquiera el fundamento paradojal de su prejuicio, al no advertir el hecho de que para Schlegel lo sensible no se refiere sólo al carácter material de los objetos del arte, sino también a la percepción misma de ellos, esto es, a la estética, adhiriendo de este modo al concepto culto derivado en la modernidad de la expresión griega Aisthesis, es decir originado en el nombre propio que denomina a los sentidos, diferenciándolos de aquello que sólo puede objetivar desde el intellectus. ${ }^{5}$

Pero la disputa no se reduce a esta cuestión conceptual o disciplinaria, sino que ella posee asimismo un cariz claramente socio-político, el que en las primeras décadas del siglo XIX alcanzará también a la filosofía, particularmente a la contraposición sincrónica entre Idealismo y Romanticismo. Pues parece evidente -así lo percibió la recepción decimonónica- que lo que Friedrich Schlegel pretende poner en peligro en la novela mediante la afirmación de la individualidad de los "amantes-amigos", en cuya relación se oscila entre "la sensualidad más desenfrenada" y "la espiritualidad más espiritual" ", es nada menos que el fundamento mismo de la institución matrimonial. Cuestión que se confirmaría plenamente cuando se advierte el sentido que posee la irónica afirmación - contenida en el fragmento 34 del Atheneo contra los esfuerzos legalistas del Estado para mantener la unidad matrimonial-, que en realidad por este

\footnotetext{
Aunque la autoría de esta recensión se atribuye a Schleiermacher y ha sido publicada bajo su nombre en la prestigiosa edición crítica liderada por Hans-Joachim Birkner (cf. Schleiermacher 1988), habría que suponer la participación en su formulación de la misma Henriette Herz, la cual explicaría el tono femenino que se cree advertir en su composición, así lo sostiene Behler en su comentario de 1992.

El término fue acuñado en 1750 por Alexander Gottlieb Baumgarten.

6 "Von der ausgelassensten Sinnlichkeit bis zur geistigsten Geistigkeit" (Cf. FSKA IV: 11).
} 
mismo motivo casi todos los matrimonios serían "sólo concubinatos" (FSKA II: 170; cf. Portales y Onetto 2005: 41).

En directa contraposición a esta skepsis con respecto a la eficacia de la formalidad jurídica y su normativa de las relaciones humanas, pueden ser leídos, según mi opinión, los pasajes de índole preceptiva contenidos en los parágrafos 161 y 162 de los Fundamentos de la filosofía del derecho de Hegel, en los que a propósito de la constitución de la eticidad (Sittlichkeit) se sostiene que "el matrimonio, como relación ética inmediata, comprende en primer lugar el momento de la convivencia natural, es decir, en cuanto relación sustancial, la vida en su totalidad, como realidad de la especie y de su proceso" (...) pero desde el punto de vista objetivo, el matrimonio sería "el libre consentimiento de las personas para renunciar a la propia personalidad natural individual y constituir la unidad en una sola persona" (Hegel 1955: 150). ${ }^{7}$

En las últimas décadas, la investigación acerca del Romanticismo ha destacado con razón, desde las lecturas interpretativas de Lucinde y de la obra fragmentaria, el carácter emancipador del pensamiento de Schlegel ${ }^{8}$, incorporándolo de lleno a la tradición liberadora que distingue en la reflexión sobre las relaciones sociales el ámbito particular de los derechos de la mujer. Su interés no se reduce, sin embargo, al aspecto sociopolítico de la emancipación femenina, aunque por cierto repara en ello, y lo hace además en tiempos en que a la mujer se la concebía, con buena conciencia y por parte de prohombres de la cultura, antes que nada como esposa y madre, relegada por lo mismo a las tareas domésticas, a la crianza, y al ejercicio de una piedad religiosa edificante inhibidora de la sexualidad. ${ }^{9}$ Pero su mirada se dirige también a la femineidad como un modo de ser humano que posee su propia estructura y que se explica a partir de sí misma. No quisiera hacer uso aquí, y en este preciso contexto, del término deconstrucción (otros ya lo han hecho) ${ }^{10}$, sobre todo porque se corre el riesgo de parecer muy apresurado por reclamar la vigencia del pensamiento de un autor decimonónico mediante su sola incorporación al significado de un léxico tan reciente y por ello estrictamente contemporáneo, pero la verdad es que su sentido se deja aplicar muy bien al procedimiento operativo que Schlegel lleva a cabo en sus

En el parágrafo 519 de la Enciclopedia de las ciencias filosóficas de 1830 se expresa esta misma cuestión de la siguiente manera: "Estas [distintas] personalidades se unen con arreglo a su singularidad exclusiva y hacen una sola persona; la intimidad subjetiva, determinada [ahora] a unidad sustancial, convierte esta unión en una relación ética, o sea, en matrimonio. La intimidad sustancial hace del matrimonio vínculo indiviso de las personas, o sea, matrimonio monogámico. La unión corporal es consecuencia del vínculo éticamente anudado".

$8 \quad$ Cf. Behler 1988: 9 y ss.; Portales 2000: 231 y ss.; y, especialmente, Rehme-Iffert 2002: 111 y ss.

$9 \quad$ No puedo detenerme aquí en el importante significado de la emancipación femenina y de la participación de mujeres al interior del movimiento romántico (remito para ello a Rehme-Iffert 2002), por lo que me limito sólo a subrayar que en este punto uno de los adversarios más importantes fue Schiller, cuyo androcentrismo fue ridiculizado constantemente, como lo muestra el siguiente ejemplo lleno de humor: Schiller escribe: “¡Honrad a las mujeres!,/ que trenzan y tejen/ rosas celestiales en la vida terrena,/ trenzan el lazo placentero del amor/ y con el honesto velo de la gracia/ alimentan vigilantes el fuego eterno/ de bellos sentimientos con mano sagrada." ("Ehret die Frauen! Sie flechten und weben/ Himmlische Rosen ins irdische Leben,/ Flechten der Liebe beglückendes Band,/ Und in der Grazie züchtigem Schleier/ Nähren sie wachsam das ewige Feuer/Schöner Gefühle mit heiliger Hand.”). Y August Wilhelm Schlegel parodia: “¡Honrad a las mujeres!,/ que calcetan las medias,/ de lana calientes para vadear los pantanos,/ remiendan calzones desgarrados,/ le hacen al marido sustanciosas sopas,/ le arreglan a los niños las lindas muñecas,/ economizan con parco salario." ("Ehret die Frauen! Sie stricken die Strümpfe,/Wollig und warm, zu durchwaten die Sümpfe, Flicken zerrissene Pantalons aus;/ Kochen dem Manne die kräftigen Suppen,/ Putzen den Kindern die niedlichen Puppen,/ Halten mit mässigem Wochengeld haus.”). Citado en Raposo y Münster 1987: XII. 
reflexiones sobre la diferencia sexual, las que suponen una autobjetivación críticoanalítica de lo masculino, una indagación sobre la composición de la masculinidad que ya no se permite registrarla simplemente como una realidad positiva cuya solvencia social y económico-política autorice a eximirla de toda elucidación ontológica. A esta operatividad deconstructiva, llama Schlegel, haciendo un juego de palabras con el título de la gran novela de Goethe, "Años de aprendizaje de la masculinidad" (Lehrjahre der Männlichkeit), la que en su expresión literaria es presentada como "una maravillosa e ingeniosamente significante alegoría de la perfección de lo masculino y lo femenino hacia una Humanidad completa y plena" que se resuelve en un androginismo lúdico o empatía sexual en el que se cambian los papeles y se rivaliza "sobre quién sabe remedar al otro más perfectamente: si a ti te sale mejor la cuidadosa vehemencia del varón o a mí la atractiva entrega de la mujer" y que identifica "el más alto grado de calidez armónica" con la situación del joven que "ya no ama solamente como un varón, sino también al mismo tiempo como una mujer" (FSKA V: 12-13 y 26).

Pero al escándalo sociopolítico de un supuesto desenfreno de la sensibilidad en desmedro de la razón y su orden moral, que caracterizó a la primera recepción de Lucinde, se añade también una segunda controversia. En ella se vislumbra ya la cuestión de la índole experimental de un escrito literario. La extrañeza provocada por la estructura de la novela se deja percibir no sólo en su tiempo, por medio de la reacción desconcertada de la recepción decimonónica, sino que en algún sentido se puede percibir además su influencia en el silencio u olvido disciplinario por parte de los estudios dedicados a Schlegel durante el siglo XX y que se extienden hasta el presente.

El término experimental, cuando referido a las artes, sugiere muy a menudo la impresión de un tipo de ensayo que contiene cierta forma de fracaso asociado a una precocidad vanguardista (que la implacable progressio de Cronos termina por relegar siempre a la retaguardia), a una antelación impaciente propia de la juventud, entendida ésta, por cierto, como etapa anterior a la madurez y a la consolidación fundada de un pensamiento o una propuesta estética, en la que los tiempos de la vida y del proceso de la creación de una obra de arte aparentan coincidir virtuosamente. En el caso de Schlegel, tal impresión podría verse confirmada por el hecho de que su obra tardía tomó más bien la forma de la reflexión filosófica y del discurso académico, desarrollándose mediante lecciones universitarias (Vorlesungen) dedicadas a los "grandes" temas de la historia y del arte. Si a esto se suma el proceso biográfico que conduce desde un joven revolucionario y rebelde hasta un viejo que ha optado por la Restauración y se ha convertido al credo del catolicismo romano sirviendo en la corte vienesa, bien se podría pensar que el valor de la obra precoz habría sido ya desestimado por su propio autor. Pero esta imagen tópica es en realidad falsa. Uno de los logros de la monumental edición crítica de las obras de Friedrich Schlegel, iniciada a mediados del siglo pasado por Ernst Behler, Jean-Jacques Anstett y Hans Eichner y que se extiende hasta el presente, es demostrar que esta presunta desvalorización de la obra temprana no sólo es inexistente, sino que por el contrario, el corpus de estos escritos manifiesta más bien que Schlegel se refirió favorablemente a su novela prácticamente en todas las etapas de su vida intelectual. ${ }^{11}$

Cf. Repertorium F. Schlegelscher Begriffe zur Lucinde, en Studienausgabe 1999: 129-211. 
Despejado este prejuicio, y atenuado en parte el daño de sus consecuencias, centro ahora la atención en la recepción temprana de la novela. Si el mayor adversario filosófico del romanticismo en general y del pensamiento de Schlegel en particular se identifica con Hegel, quien denunció incansablemente, y por raison d'État, el poder corrosivo de la ironía romántica en relación a las primeras proposiciones estéticas, la principal crítica provino desde la poesía clásica de Weimar, en la obra de Schiller. ${ }^{12}$ Tal vez el testimonio más interesante se encuentre en algunas expresiones privadas de Schiller, contenidas en una carta dirigida a Goethe, fechada en Jena el 19 de junio de 1799 (Staiger 1987: 781-782). Allí relata el efecto que ha producido en él la lectura de Lucinde, advirtiendo de entrada que su primera reacción ha sido una irritante cefalea que aún perdura al momento de escribir la carta. La novela retrataría fielmente el carácter grotesco o ridículo de su autor y su estructura manifestaría algo "eternamente informe y fragmentario, y un emparejamiento altamente extraño de lo nebuloso con lo característico". ${ }^{13}$ Más adelante, se lamenta Schiller del malestar que le produce esa "charla vacía", por lo que se niega a leer el texto hasta el final y concluye así el párrafo de la carta: "Después de la fanfarronada de la grecomanía y después de la época que Schlegel dedicó al estudio de la misma, yo hubiera esperado que se me hiciera recordar un poco la simplicidad e ingenuidad de los antiguos, pero este escrito es el colmo de la anti-forma y de la anti-naturalidad modernas; uno cree leer una mezcolanza de Woldemar, de Sternbald y de una descarada novela francesa". ${ }^{14}$

Fragmentariedad e inconclusión, falta de forma y de naturalidad, sin sentido, sincretismo grotesco de estilos y épocas, además de una mezcla arbitraria de productos de la modernidad literaria: estos son los conceptos que describen, según Schiller, la estructura de Lucinde y los que la convierten en una obra ilegible. Creo que el listado refleja una reacción muy típica de la crítica que se enfrenta a lo que habitualmente se llama literatura experimental, pues frente a la complejidad de su objeto ella se queda sin una pauta clara de análisis y opta por declarar la incomprensibilidad del texto en cuestión.

Por este motivo, me parece que una desprejuiciada aproximación interpretativa de un escrito de esta índole debe buscar los criterios analíticos en la obra misma que pretende dilucidar, sobre todo porque si algo caracteriza en su intimidad a la producción experimental, es que ella muestra siempre las herramientas involucradas en su elaboración, transparentando sin pudor el artificio del deus ex machina, dejando ver intencionalmente la tramoya que sostiene a la ficción.

En primer lugar, este modo de proceder debe reconocer el contexto filosóficoestético en que se produce la novela, advirtiendo su lugar dentro de los marcos del proyecto romántico de una progressive-universal-Poesie en la que, como ya se ha insinuado anteriormente, desaparecen los rígidos límites de la separación de los

Para la crítica hegeliana del romanticismo, cf. Hegels Kritik der Romantik (Pöggeler 1999, especialmente p. 121 145). Las objeciones provenientes de Schiller se refieren sólo a la primera época, pues el poeta falleció el año 1805.

13 “...hier ist das ewig Formolse und Fragmentarische und eine höchst seltsame Paarung des Nebulistischen mit dem Charakteristischen"

14 Se refiere a las novelas de Friedrich Heinrich Jacobi y Ludwig Tieck. Para la traducción de la expresión coloquial arcaica nach den Rodomontaden von Griechheit he optado por la versión después de la fanfarronada de la grecomanía de Berta Raposo (Cf. Raposo y Münster 1987: I). 
géneros literarios. El sincretismo deja de ser así un defecto de promiscuidad y se transforma en una exigencia estética original. A esto se une el inicio poiético que posee su punto de partida en lo que Schlegel denomina nostalgia de infinitud, la que determina la tarea creativa como un proceso interminabilis, por lo que también la fragmentariedad pierde su atributo de mera incapacidad de concluir y se emparenta más bien con el devenir siempre fluyente de las aguas que conforman el río perpetuamente cambiante cuya única identidad consiste en ser siempre distinto de sí mimo, metáfora que la tradición doxográfica atribuye a Heráclito de Éfeso, llamado el Oscuro.

Por otra parte, el carácter experimental se hace también patente, a mi juicio, en el hecho de que el autor se permite una intromisión metodológica, por decirlo de alguna manera, en el acontecimiento propiamente literario del relato. De este modo, el lector de Lucinde se entera que se enfrenta a una estética de lo bello, pues el mundo no es percibido mediante un cálculo de probabilidades que concluya en su tendencia a la perfección, como pretendía Leibniz con su aforismo apologético del Essai de Théodicée, sino que se constata más bien que "aunque el mundo no sea precisamente el mejor o el más práctico, sin embargo sé que es el más bello" (FSKA $\mathrm{V}$ : 5). La aproximación poética a dicho objeto requiere de vías de acceso que la misma literatura ya ha sugerido, cuya filiación estética se menciona en el prólogo: los nombres de Petrarca, Bocaccio y Cervantes expresan los principales antecedentes que debiesen orientar al lector en la comprensión de la estructura de la novela en dependencia epigonal (FSKA V: 3 ).

El primero de ellos se identifica con el giro moderno de la poesía que emerge desde la superación de la época oscura, un nuevo comienzo que supone un renacimiento fomentado por la reconciliación de la herencia moderna del cristianismo de la antigüedad tardía con la Grecia clásica. ${ }^{15}$ Los otros dos expresan, en sus obras Decameron y Don Quijote, una literatura conformada por un concierto discursivo intencional de diversos géneros retóricos, en una manera escritural que Schlegel concibe como una estética del arabesco. Concepto que el escrito Brief über den Roman de 1800 define mediante la expresión: "forma determinada y esencial del modo de exteriorización de la poesía" ${ }^{16}$, y que la Rede über die Mythologie percibe como una creación artística semejante al "ingenio (Witz) de la poesía romántica", advirtiendo que ella no consiste en "ocurrencias singulares, sino en la construcción del conjunto", tal como se muestra "una y otra vez en las obras de Cervantes". El párrafo prosigue describiendo un "desorden artísticamente ordenado" (künstlich geordnete Verwirrung), una "fascinante simetría de contradicciones", una "maravillosa eterna alternancia de entusiasmo e ironía, que vive ella misma en las más pequeñas partículas del todo", y concluye con la afirmación: "el arabesco es la forma más antigua y original de la fantasía humana”. (FSKA II: 318-319).

15 Aunque el movimiento romántico no aceptó totalmente la tesis ilustrada que atribuía un período de oscuridad a toda la así llamada Edad Media y fue rescatando paulatinamente sus contenidos para la historia de la literatura romántica (véase el magnífico trabajo Die Mittelalter-Rezeption der Brüder Schlegel; Höltenschmidt 2000), la identificación con la modernidad literaria renacentista es indiscutible. Así lo expresa, por ejemplo, la obra Geschichte der romantischen Literatur de August Wilhem Schlegel (cf. Schlegel 1965), en la que luego de revisar sucintamente algunos aspecto de la mitología medieval, comienza directamente con Dante, Petrarca y Bocaccio.

16 “...ich halte die Arabeske für eine ganz bestimmte und wesentliche Form der Außerungsart der Poesie” (FSKA II 330). 
La alusión a la estética del arabesco, cuyo significado había sido objeto de discusión en los círculos literarios de la época y defendida por Goethe en su Von Arabesken de 1789, es de gran importancia para poder comprender la compleja estructura de este ensayo literario. La analogía de estos disímiles productos de la creación artística podría expresarse de la siguiente manera: así como el arabesco produce la sensación visual de una fantástica afinidad espacial mediante una geometría del desorden alcanzada por la ornamentación pictórica de líneas anacolutas y de figuras particulares inconexas, así también Lucinde se desenvuelve por medio de ditirambos, alegorías, cartas, confesiones, metamorfosis, reflexiones y jugueteos de la fantasía para culminar en la novela romántica.

Se trata pues de una armonía estética que sólo puede proceder del caos. ${ }^{17} \mathrm{El}$ tono poético se ve obligado a sintonizar con una pluralidad de sentidos y dejar que la belleza surja de esta vorágine de direcciones opuestas e incluso contradictorias. Romantizar el mundo se manifiesta así como lo contrario de la búsqueda de un orden original de las causas, propia de la teleología, su cometido se encuentra muy lejos de ese discurso que consiste en "clasificar y descomponer conceptos" y se permite incluso operar desde el "olvido de las reglas de la razón, la moralidad y el transcurso del tiempo" (FSKA V: 8). Sin duda que la lectura, educada en la pulcritud de la formalidad clásica, acostumbrada al reconocimiento de aquello que de alguna manera ya sabe, y frente a lo cual posee de antemano los criterios de su valoración, se siente incómoda con una obra de este tipo, y preferiría por cierto la compañía de los discursos sobre el Eros, contenidos en el Banquete de Platón, que a Eros mismo devenido discurso, como lo intenta el texto de Lucinde.

Tal vez haya, sin embargo, algo de verdad en el tópico que proclama el congénito fracaso de la literatura experimental, pero habría que añadir que en ocasiones este fracaso es intencional, o al menos que no se está dispuesto a evadirlo, en la medida en que se entiende como destino propio de toda particularidad de obra, sentenciada a dispersarse en el flujo de la progressive-universal-Poesie. Por otra parte, el fracaso no siempre se encuentra del lado de la obra misma, no siempre atañe a la escritura, sino también a la lectura. Pienso, por ejemplo, en el más paradigmático de los ensayos de experimentación literaria en que la armonía estética deviene a partir del caos, me refiero a ese intento de monumentalidad homérica en que una polifonía de voces, pensamientos, murmullos, cantos, sonidos, olores, acontecimientos domésticos y un extendido etcétera, se reúnen en la unidad casi invisible de la efeméride espacio temporal de la ciudad de Dublín el día jueves 16 de junio de 1904. Tal es el acontecimiento único y universal del Ulises de James Joyce, en donde muy a menudo se advierte que el fracaso no está en la obra, sino en la vivencia del lector, del mismo modo como Lucinde parece haber provocado una suerte de analfabetismo en Schiller.

La historia de la recepción señala, no obstante, que el Lucinde ha tenido un escaso eco en la reflexión estética o en la teoría literaria de la novela moderna. Su estructura experimental no ha sido objeto de estudios específicos, ni tampoco ha producido epígonos vanguardistas. Su importancia se refleja, empero, en los múltiples trabajos que la obra ha inspirado dentro de los marcos de la investigación sobre la génesis y primer desarrollo del Romanticismo. En ellos se destaca, sobre todo, el hecho de

Casi todas las alusiones a Lucinde contenidas en la obra fragmentaria, ya sea en Fragmente zur Poesie (FSKA XVI) o Philosophische Lehrjare (FSKA XVIII) la vinculan con el origen caótico. 
proponer una literatura sin autor, o si se quiere, en donde la autoría queda en manos del texto mismo mediante una contraposición de voces polifónicas. Por otra parte, su insistencia en transgredir los límites de los géneros canónicos se funda en una nueva teoría de la subjetividad, en la que la escritura no acepta ya la distinción entre lo que es objeto de la estética y aquello que se considera propio de la reflexión filosófica. La mención de estos dos aspectos característicos de la producción literaria del Romanticismo sugieren la vigencia de sus propuestas en los tiempos que se identifica con la modernidad tardía.

\section{OBRAS CITADAS}

Behler, Ernst y Jochen Hörisch. 1987. Die Aktualität der Frühromantik. Paderborn: Schöningh.

Behler, Ernst. 1988. Studien zur Romantik und zur idealistischen Philosophie. Paderborn: Schöningh.

Behler, Ernst. 1992. Friedrich Schlegel: Lucinde. En: Interpretationen Romane des 19 Jahrhunderts. Stuttgart: Reclam.

Behler, Ernst. 1996. Friedrich Schlegel. Hamburg: Rowohlt.

Dilthey, Wilhelm. 1970. Leben Schlaiermachers. Erster Band. Gesammelte Schriften XIII. Göttingen: Vandenhoeck \& Ruprecht.

Holmes, Sussane. 2006. Synthesis der Vielheit. Die Begründung der Gattungstheorie bie August Wilhelm Schlegel. München: Schöningh.

Höltenschmidt, Edith. 2000. Die Mittelalter-Rezeption der Brüder Schlegel. Paderborn: Schöningh.

Pöggeler, Otto. 1999. Hegels Kritik der Romantik. München: Wilhelm Fink.

Portales, Gonzalo. 2000. "Dialécticas del dolor. En torno a Hegel y el romanticismo". Seminarios de filosofia 12-13: 231-247.

Portales, Gonzalo y Breno Onetto. 2005. Poética de la infinitud. Ensayos sobre el romanticismo alemán. Santiago de Chile: Intemperie / Palinodia.

Rehme-Iffert, Birgit. 2002. "Friedrich Schlegel über Emanzipation, Liebe und Ehe”. Athenäum 12: $111-132$.

Schlegel, August Wilhem. 1965. Geschichte der romantischen Literatur. Stuttgart: Kohlhammer.

Schlegel, Friedrich. 1959 y ss. Friedrich Schlegel Kritische Ausgabe seiner Werke (=FSKA). Paderborn: Schöningh

Schlegel Schlegel, Friedrich. 1999. Lucinde. Ein Roman. Edición crítica de Karl Konrad Polheim (=Studienausgabe). Stuttgart: Reclam.

Schlegel, Friedrich. 1987. Lucinde. Traducción y notas de Berta Raposo. Introducción de Reinhold Münster (= Raposo y Münster). Valencia: Natán.

Schleiermacher, Friedrich Daniel Ernst. 1988. Vertraute Briefe über Friedrich Schlegels Lucinde Vertraute Briefe über Friedrich Schlegels Lucinde. Berlín/New York: Walter de Gruyter.

Schulz, Gerhard. 1983. Die deutsche Literatur zwischen Französicher Revolution und Restauration 1789-1830. München: Beck.

Staiger, Emil (ed.) 1987. Der Briefwechsel zwischen Schiller und Goethe. Frankfurt am Main: Insel.

Zimmermann, Harro. 2009. Friedrich Schlegel oder Die Sehnsucht nach Deutschland. Paderborn: Schöningh. 
